Extended Data for

\title{
Dynamics of Intermittent Delayed Emission in Single CdSe/CdS Quantum Dots
}

Stijn O.M. Hinterding ${ }^{1,2}$, Sander J.W. Vonk ${ }^{1,2}$, Elleke J. van Harten ${ }^{1}$, Freddy T. Rabouw ${ }^{1,2, *}$

${ }^{1}$ Soft Condensed Matter and ${ }^{2}$ Inorganic Chemistry and Catalysis, Debye Institute for Nanomaterials Science, Utrecht University, P.O. Box 80000, 3508 TA Utrecht, The Netherlands

* Corresponding author. E-mail: f.t.rabouw@uu.nl 
Figure E1-E10 - Results of double-APD experiments for more quantum dots.

Below we provide an overview of results from the double-APD (in Hanbury Brown-Twiss configuration) experiments. After each of these experiments, the same individual quantum dots were studied by a single APD and a spectrometer simultaneously (as described in the main text). For these results, see Figure E11-20.

The arrangement of each of these figures is as follows: (A) Total photoluminescence (PL) intensity $\left(I_{\text {tot }}\right)$ as a function of time. (B) Distribution of PL intensity values. The red dotted line (at $\left.I_{\mathrm{ON}}^{*}\right)$ in $(\mathrm{A}, \mathrm{B})$ indicates the threshold used to distinguish the ON state. (C) Delayed PL intensity $\left(I_{\text {del }}\right)$, defined as the number of photons detected $\geq 200 \mathrm{~ns}$ after a laser pulse. (D) Distribution of $I_{\mathrm{del}}$. (E) Fluorescence-lifetime-intensity distribution, obtained from the PL intensity and average photon delay time for each individual $10 \mathrm{~ms}$ period in the experiment. (F) Normalized second-order correlation function. Light blue line is experimental data, dark blue line is a fit to a bi-exponential model. The small area of the zero-delay peak, relative to the side-peaks, indicates a single QD was studied. (G) Background-subtracted PL decay curves corresponding to the (green) ON and (red) GRAY states. Symbols are data points, solid lines are fits to a bi-exponential decay function. Time periods were categorized as GRAY when the intensity was in the range $I_{\mathrm{GRAY}}^{*}$. (H) The same data as in $(\mathrm{G})$, now displayed on double-logarithmic axes. (I) 2-dimensional histogram of the correlation between $I_{\mathrm{del}}$ and $I_{\mathrm{tot}}$, constructed from the total double-APD experiment, binned at $10 \mathrm{~ms}$. (J) 2-dimensional histogram of the correlation between $I_{\mathrm{del}}$ and the following change in $I_{\mathrm{tot}}$, i.e. of $\left\{I_{\mathrm{del}, i}, I_{\mathrm{tot}, i+1}-I_{\mathrm{tot}, i}\right\}$. (K) Same as $\mathbf{J}$, but now correlating $I_{\mathrm{del}}$ and the preceeding change in $I_{\mathrm{tot}}$, i.e., histogramming $\left\{I_{\mathrm{del}, i}, I_{\mathrm{tot}, i}-I_{\mathrm{tot}, i-1}\right\}$. (L) Distributions of $I_{\mathrm{del}}$, corresponding to (blue) all 10-ms time bins during which the QD is ON ( $I_{\text {tot }} \geq I_{\mathrm{ON}}^{*}$ ), (red) bins directly preceding an ON $\rightarrow$ GRAY switch (i.e., selecting all bins $i$ for which $I_{\mathrm{tot}, i+1}-I_{\mathrm{tot}, i} \leq-70 \mathrm{cts} / 10 \mathrm{~ms}$ ), and (green) bins directly following a GRAY $\rightarrow$ ON switch (i.e., $I_{\mathrm{tot}, i}-I_{\mathrm{tot}, i-1} \geq 70$ cts/10 ms). Here, $I_{\mathrm{ON}}^{*}$, and $I_{\mathrm{GRAY}}^{*}$ are the ON-state threshold and GRAY-state intensity-range, respectively. The values of these parameters, as well as the estimated number of photons absorbed per laser pulse, $p \sigma$ (determined from the laser power used in the experiment, see Section S5), are provided in the following table:

\section{Table E1 - Overview of analysis and acquisition parameters of double-APD experiments}

\begin{tabular}{cccc} 
QD number & $I_{\text {ON }}^{*}(\mathrm{cts} / 10 \mathrm{~ms})$ & $I_{\mathrm{GRAY}}^{*}(\mathrm{cts} / 10 \mathrm{~ms})$ & $p \sigma$ \\
\hline 1 & 100 & {$[25,60]$} & 0.3 \\
2 & 130 & {$[35,60]$} & 0.3 \\
3 & 110 & {$[15,50]$} & 0.3 \\
4 & 110 & {$[25,55]$} & 0.3 \\
5 & 230 & {$[50,10,40]$} & 1 \\
6 & 230 & {$[70,120]$} & 1 \\
7 & 230 & {$[50,85]$} & 1 \\
8 & 220 & {$[40,85]$} & 1 \\
9 & 210 & {$[60,120]$} & 1 \\
10 & 300 & &
\end{tabular}




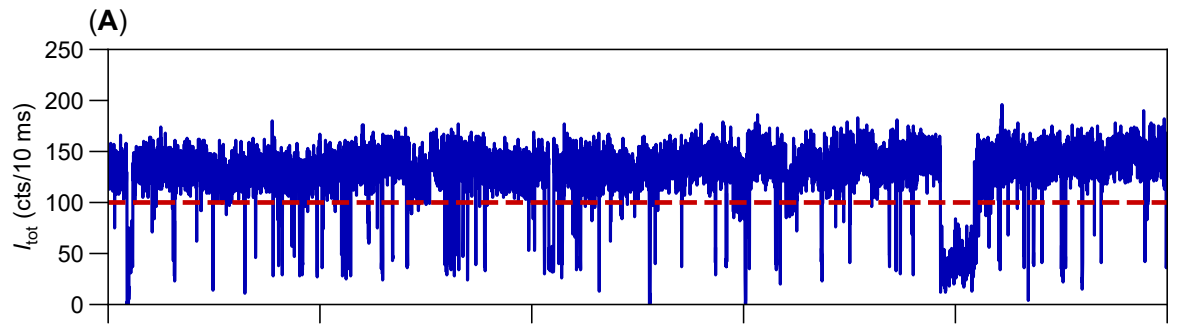

(B)

(C)

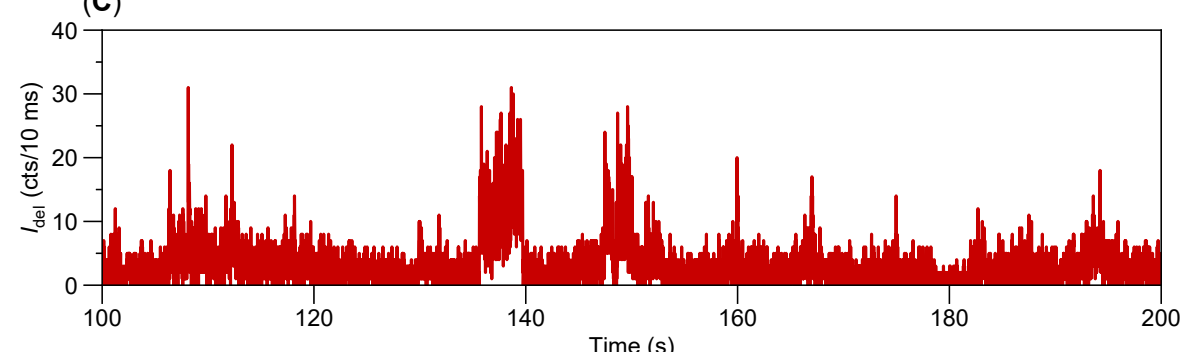

(D)

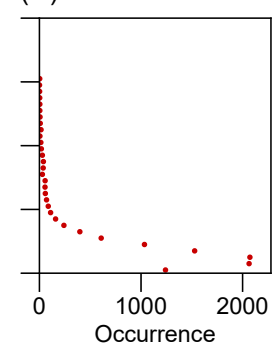

(E)
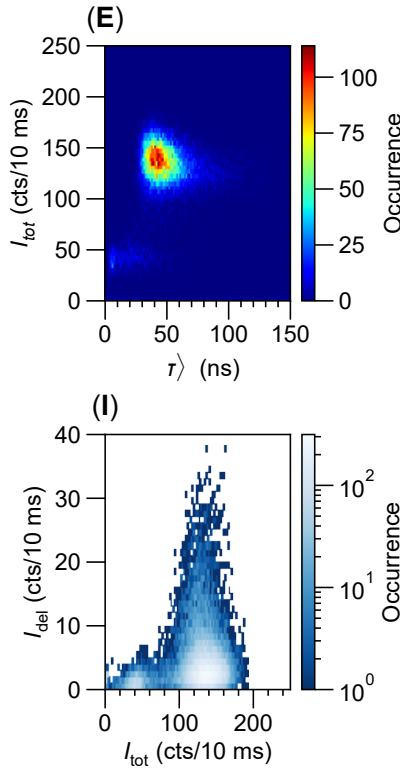

(F)

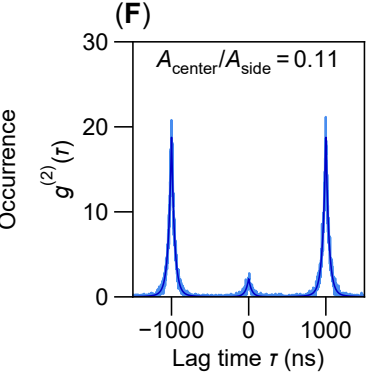

$(\mathrm{J})$

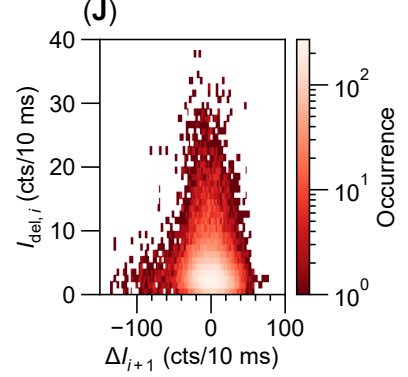

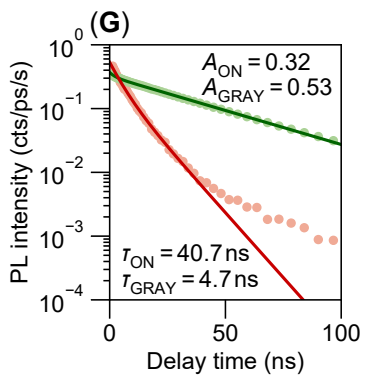

(K)

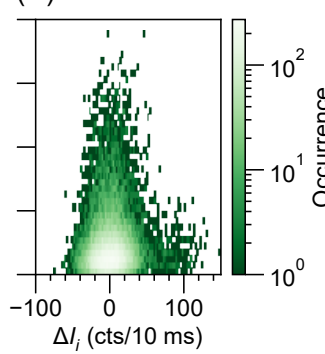

(H)

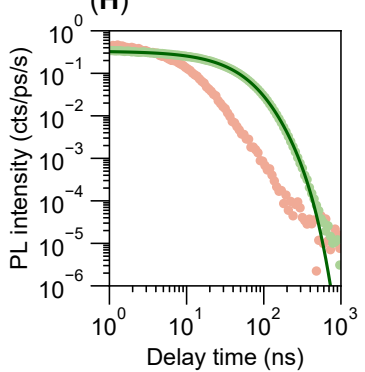

(L)

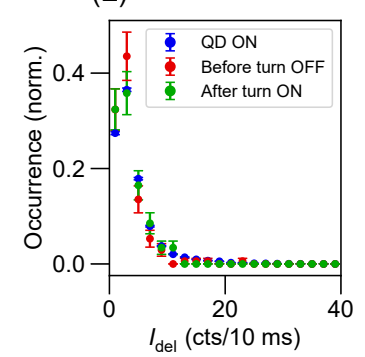

Figure E1 - Overview of photoluminescence properties of quantum dot 1 (same quantum dot as discussed in main text). 


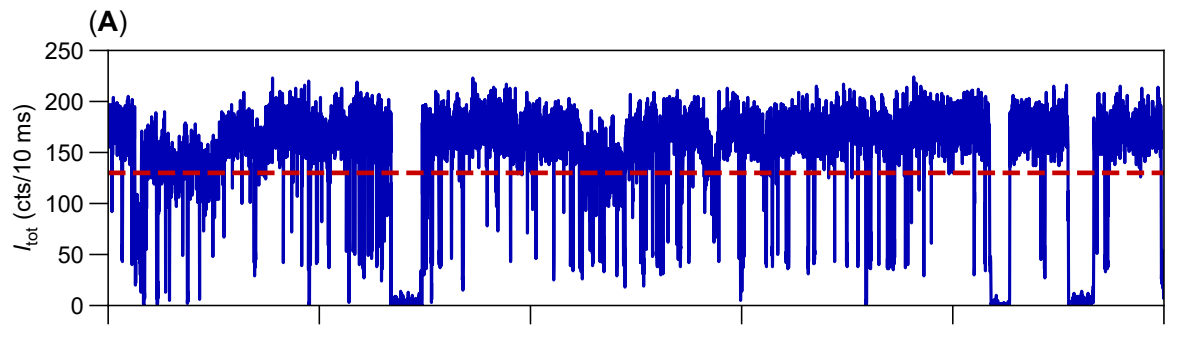

(B)

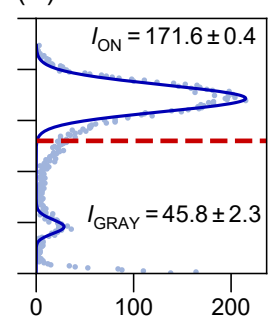

(C)

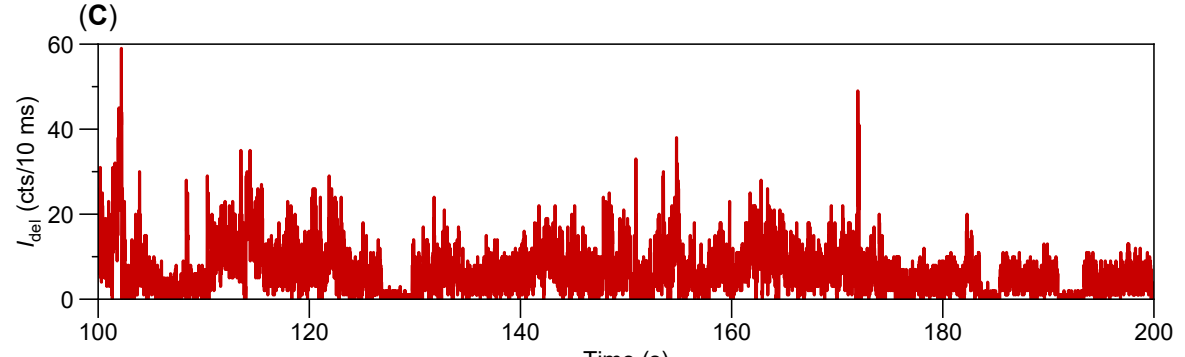

(D)

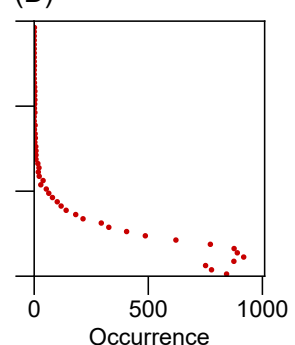

(E)
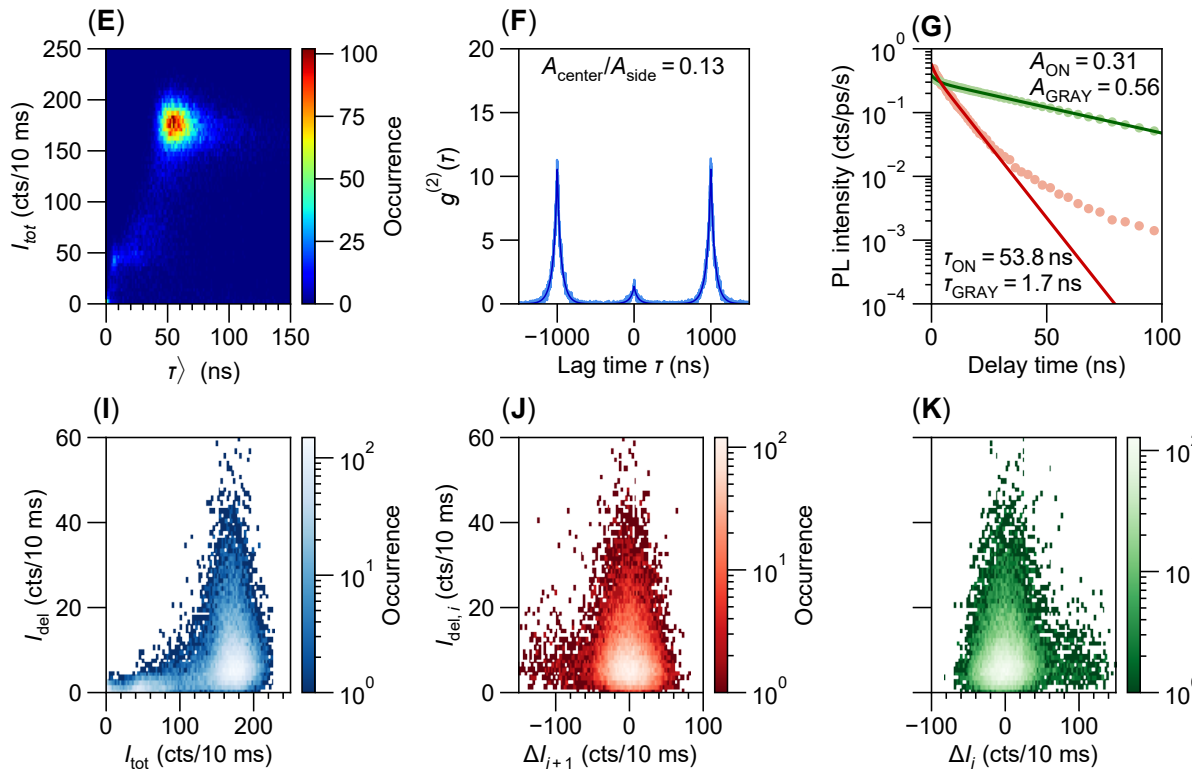

(K)
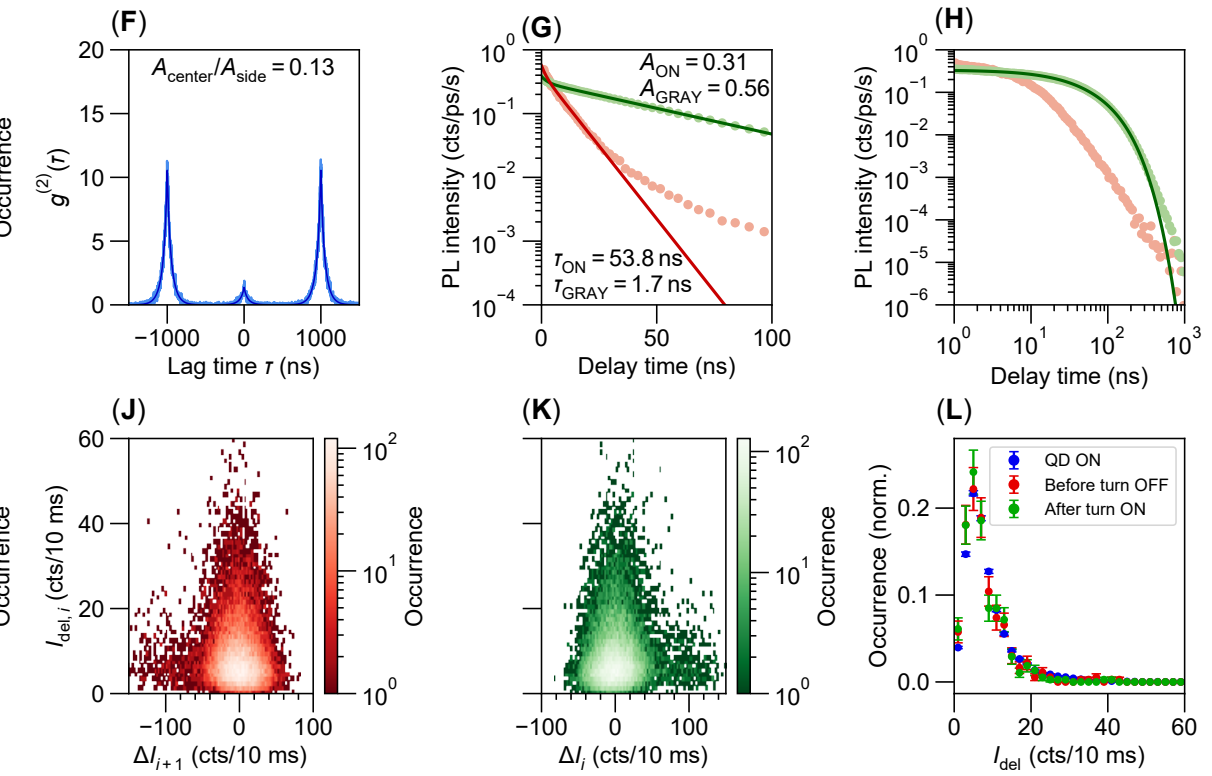

(L)

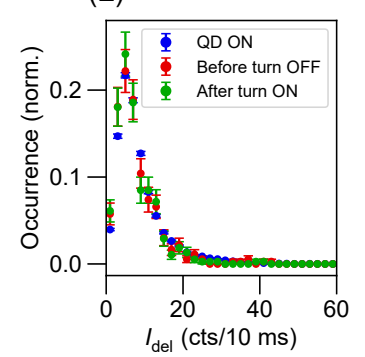

Figure E2 - Overview of photoluminescence properties of quantum dot 2. 


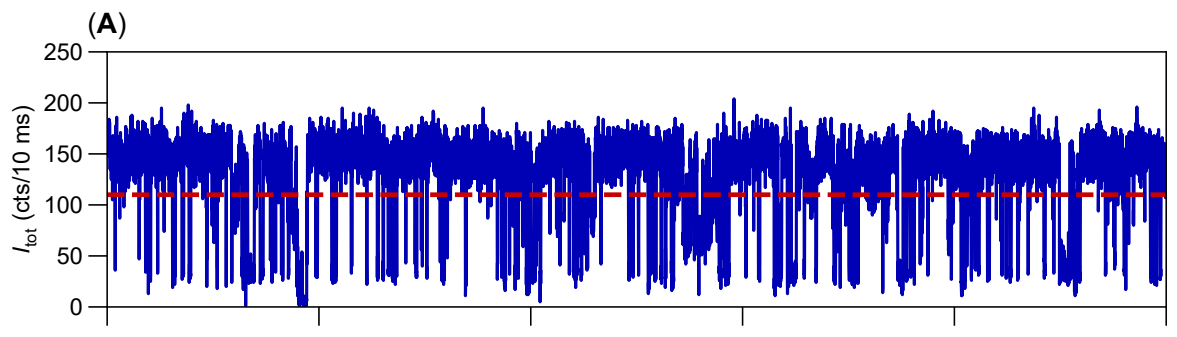

(B)

(C)

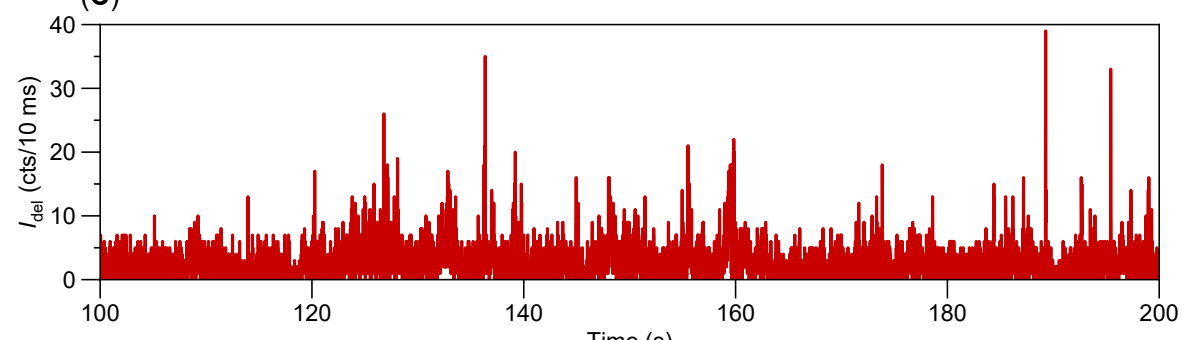

(D)

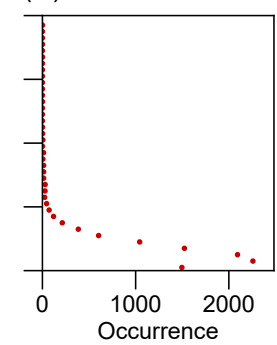

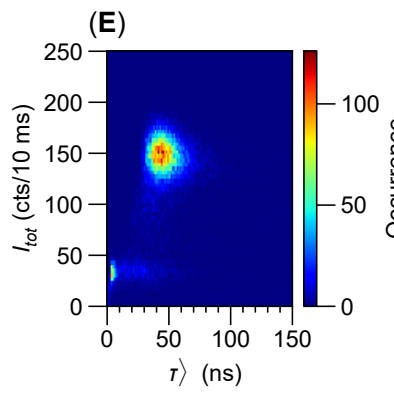

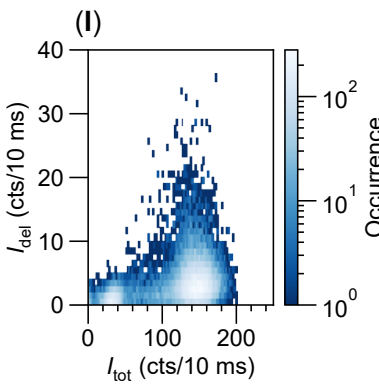

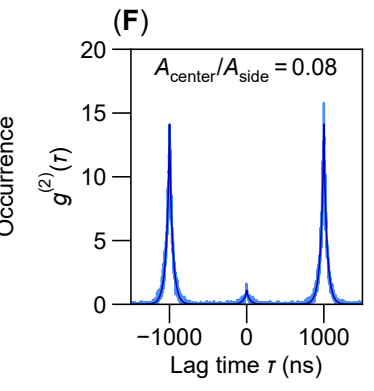

(J)

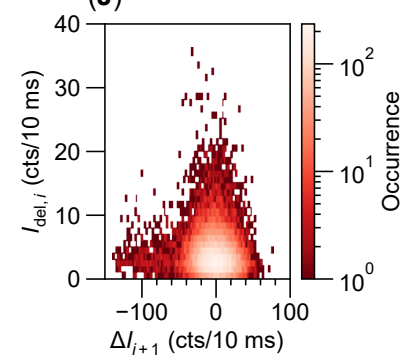

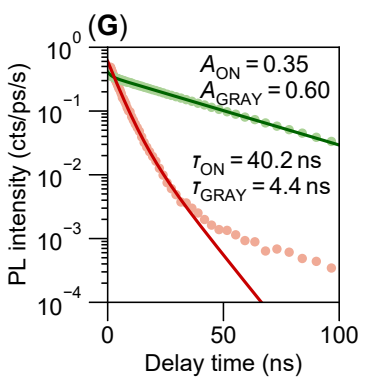

(K)

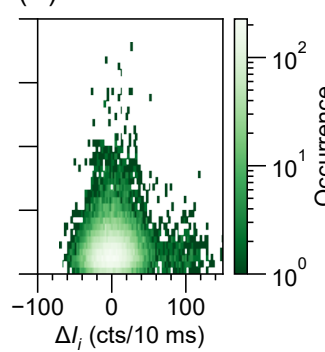

(H)

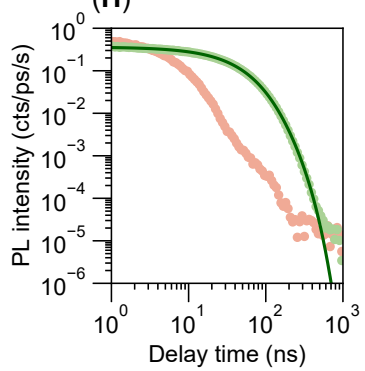

(L)

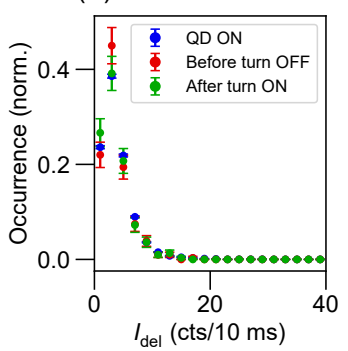

Figure E3 - Overview of photoluminescence properties of quantum dot 3. 


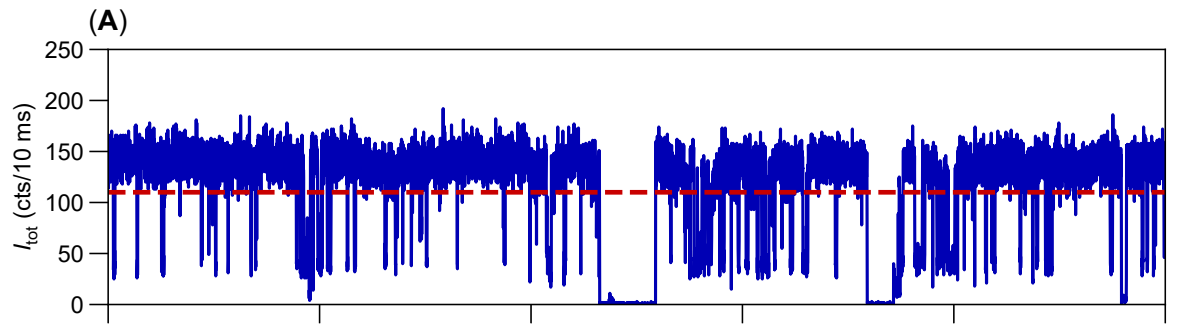

(B)

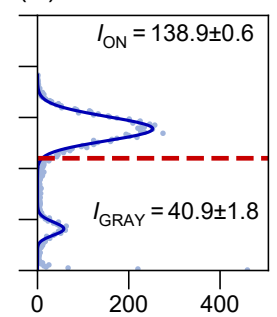

(D)
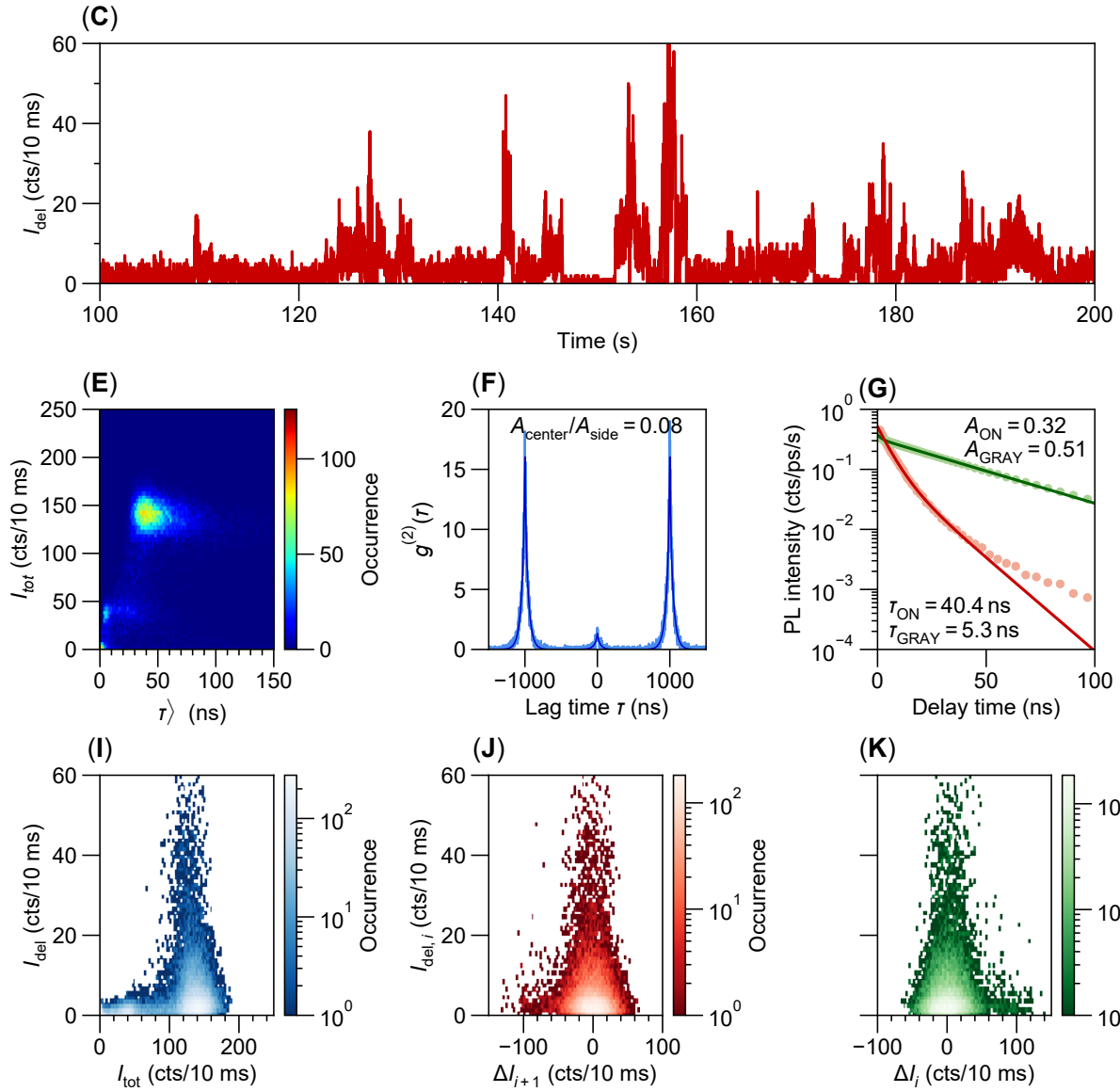

(K)

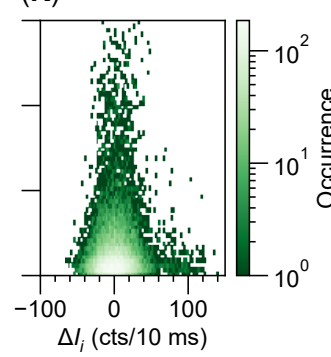

(L)
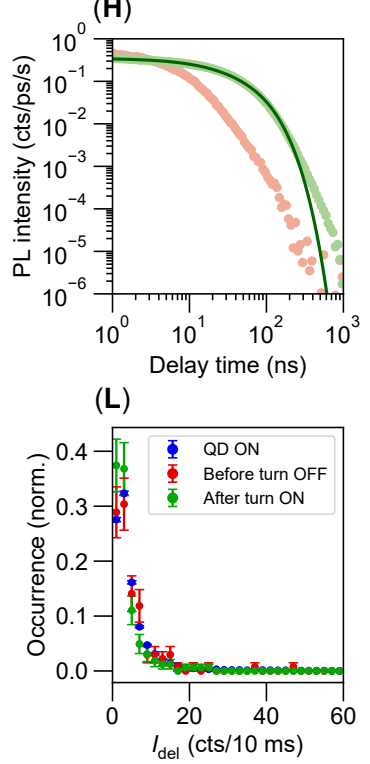

Figure E4 - Overview of photoluminescence properties of quantum dot 4 . 


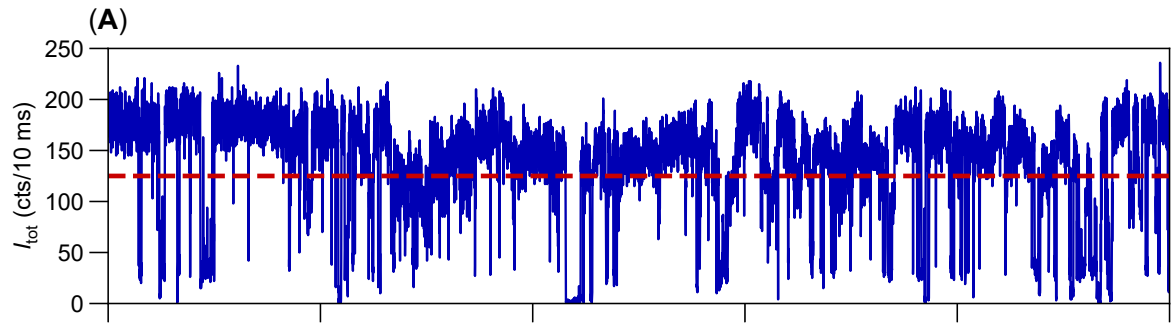

(B)

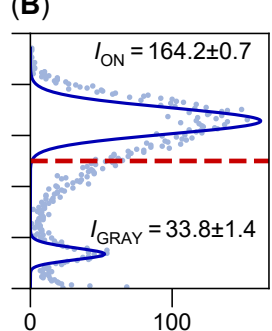

(C)

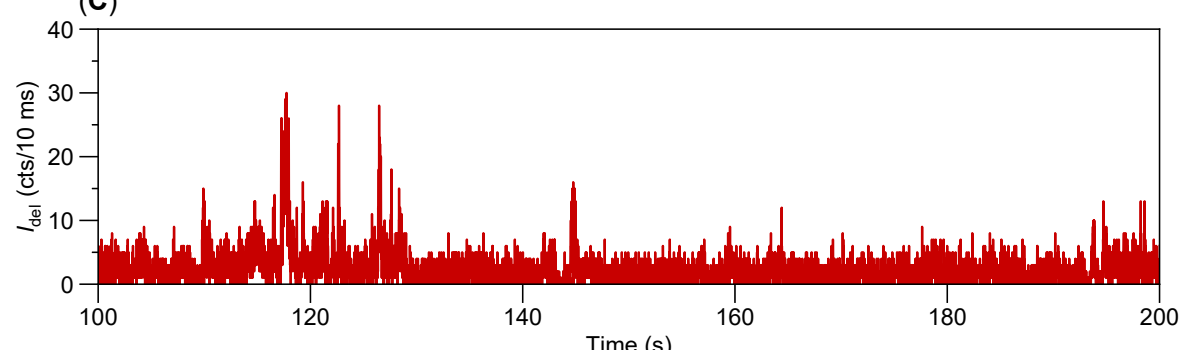

(D)

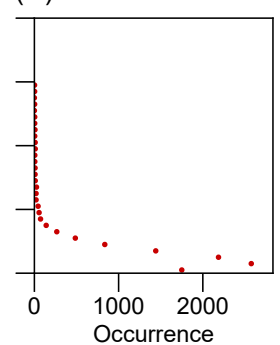

(E)
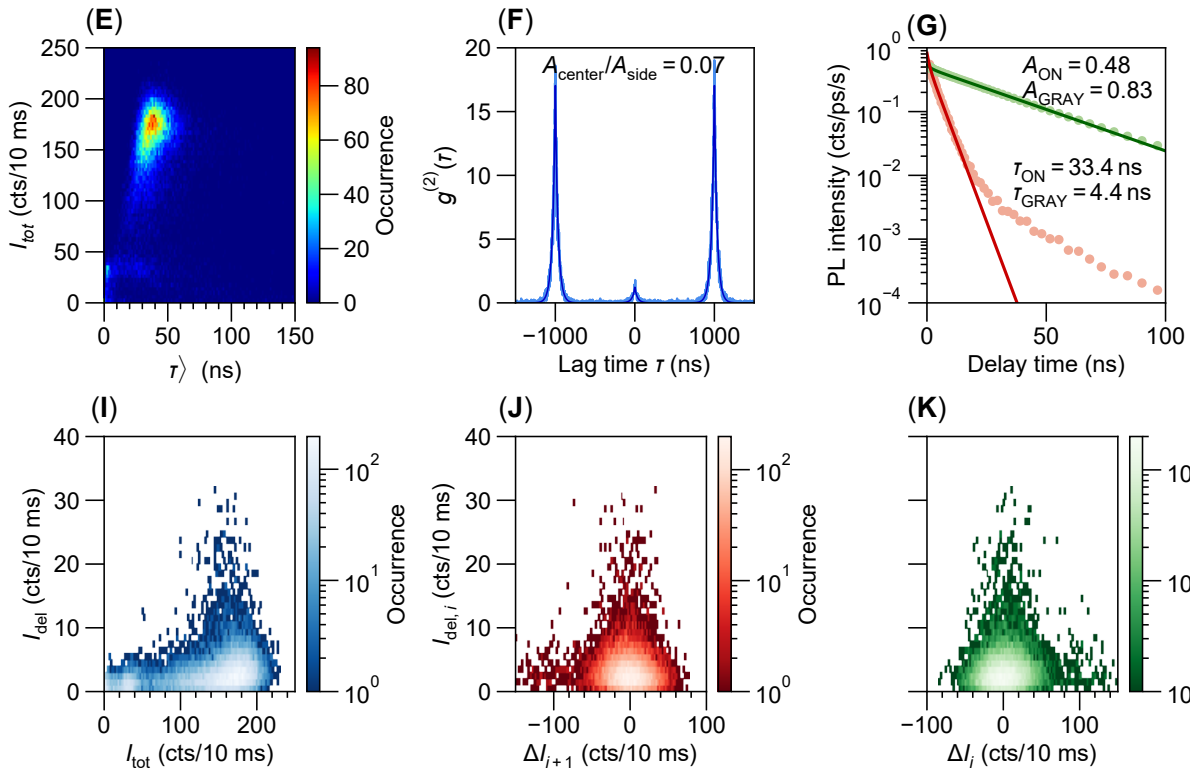

(K)

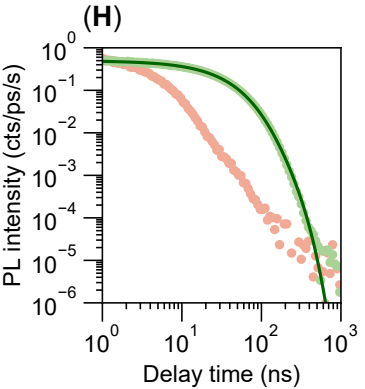

(L)
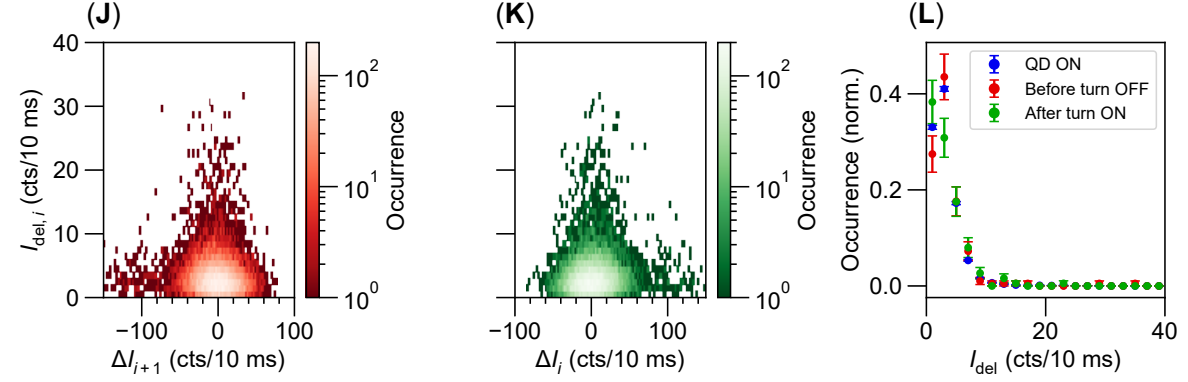

Figure E5 - Overview of photoluminescence properties of quantum dot 5. 


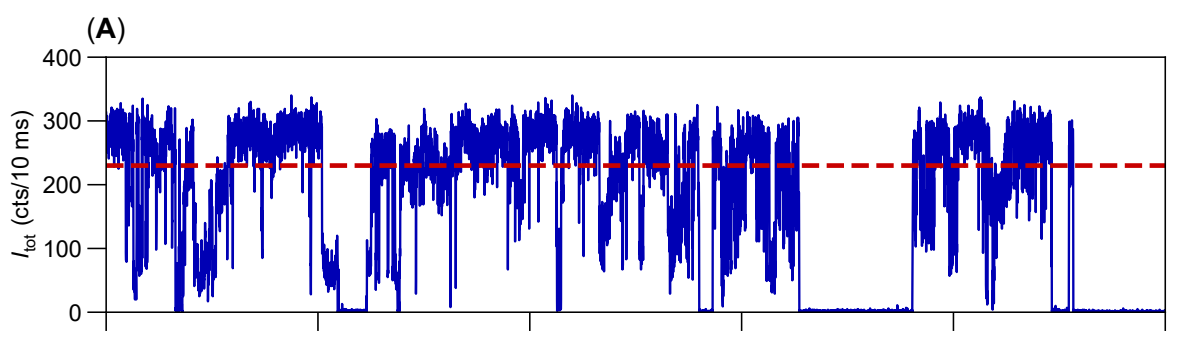

(B)

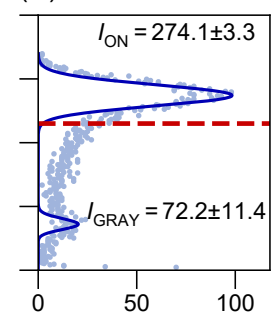

(D)

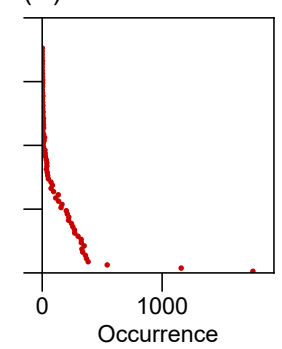

(H)

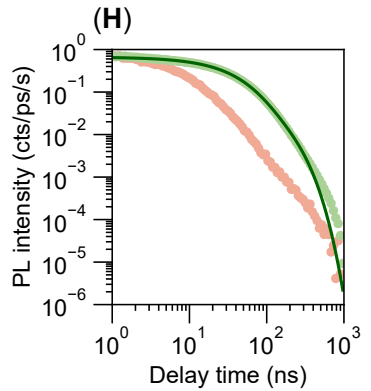

(L)
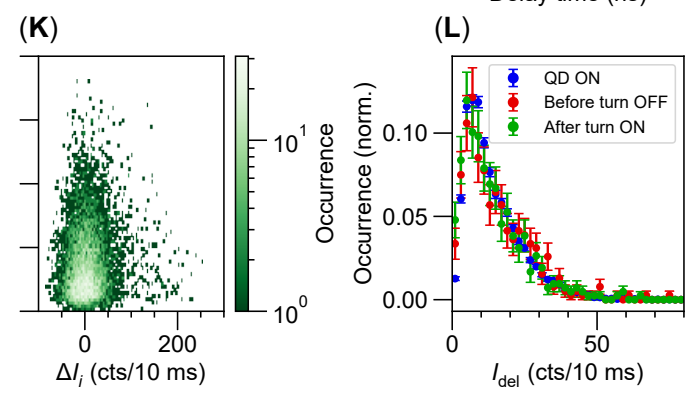

Figure E6 - Overview of photoluminescence properties of quantum dot 6. 


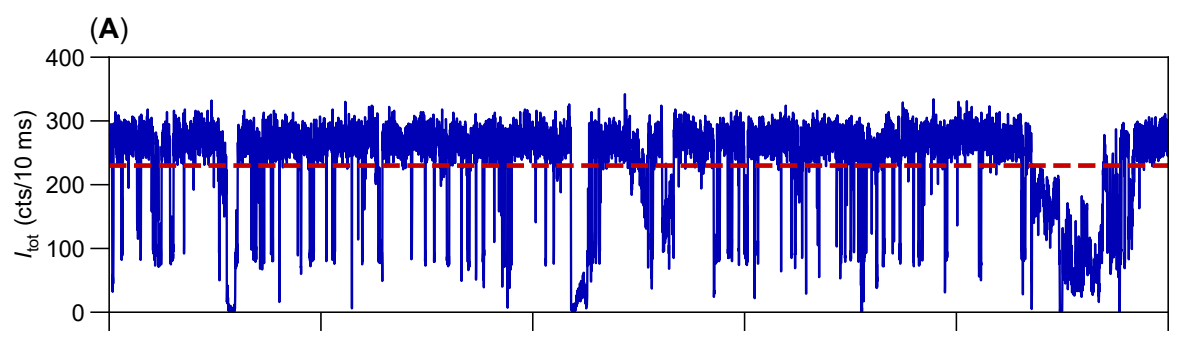

(B)

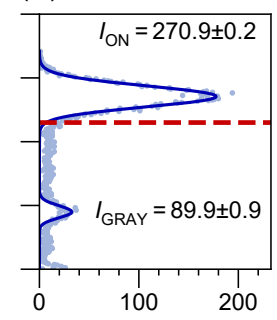

(D)

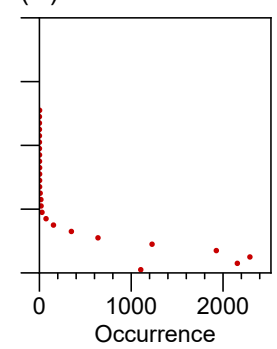

(H)

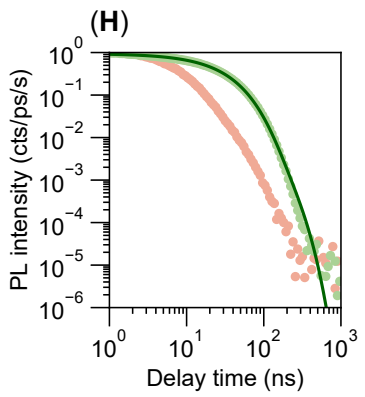

(L)

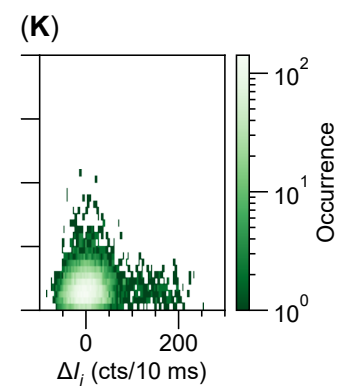

(L)

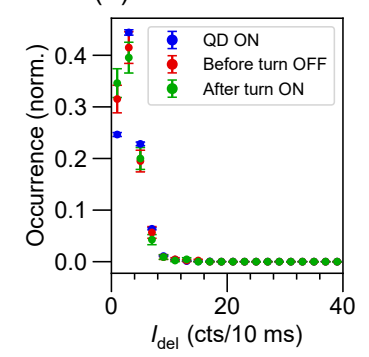

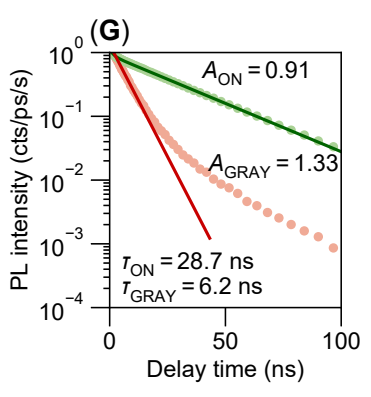

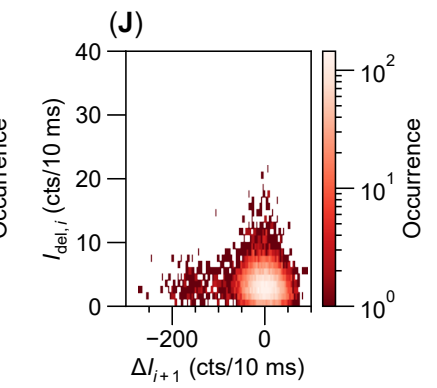

Figure E7 - Overview of photoluminescence properties of quantum dot 7. 


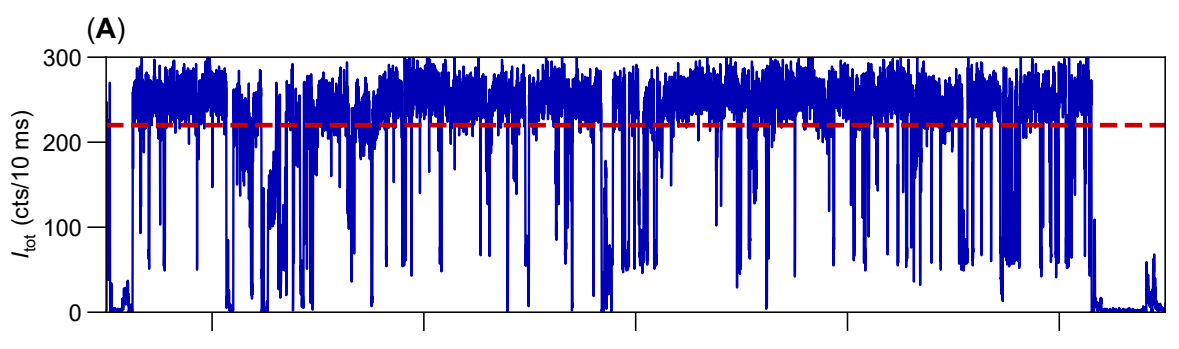

(B)

(C)

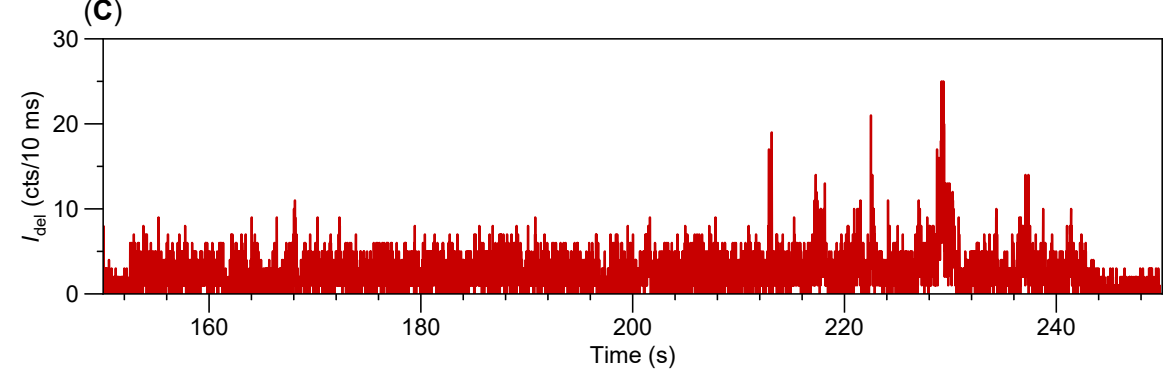

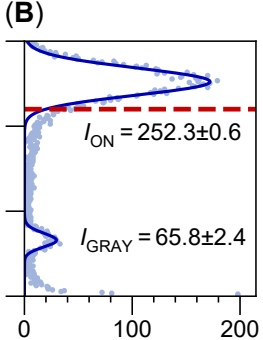

(D)
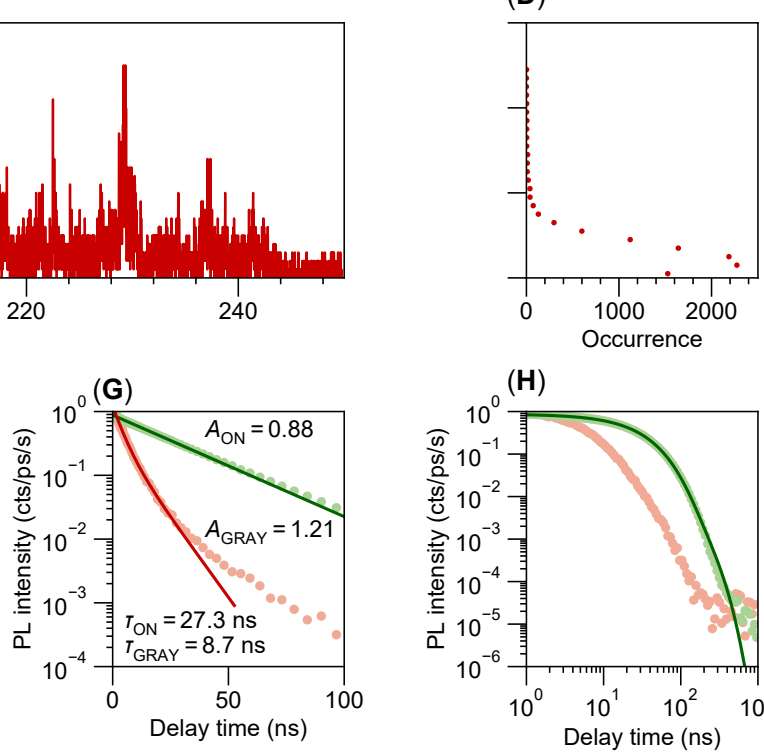

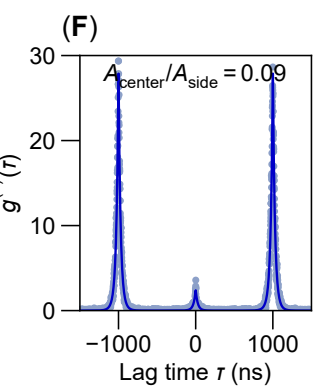

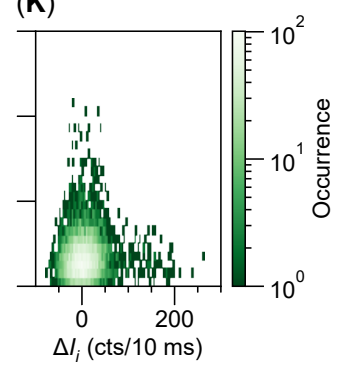

(H)

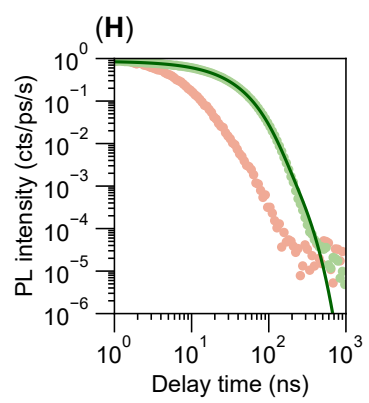

(L)

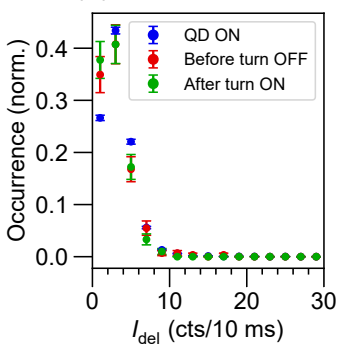

(K)
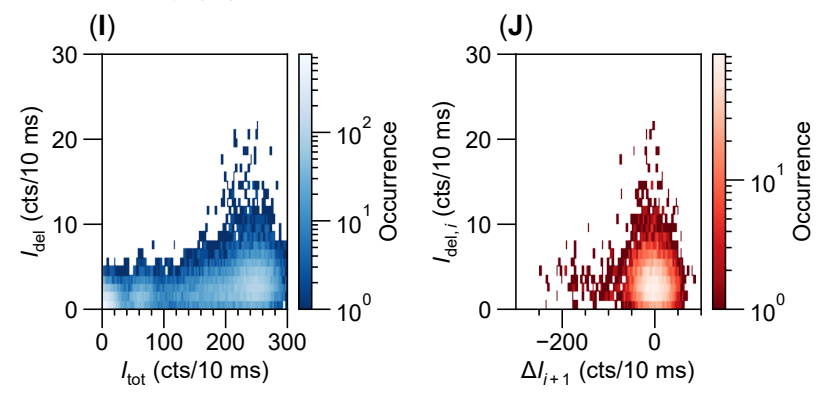

Figure E8 - Overview of photoluminescence properties of quantum dot 8. 


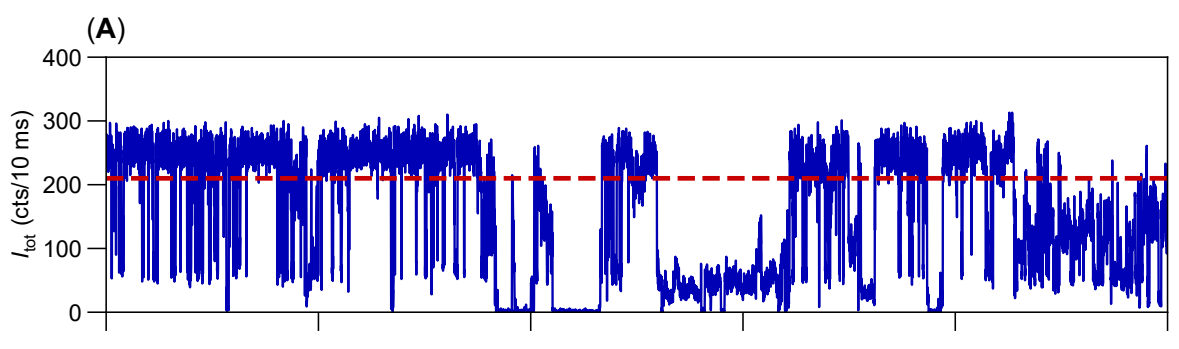

(B)

(C)

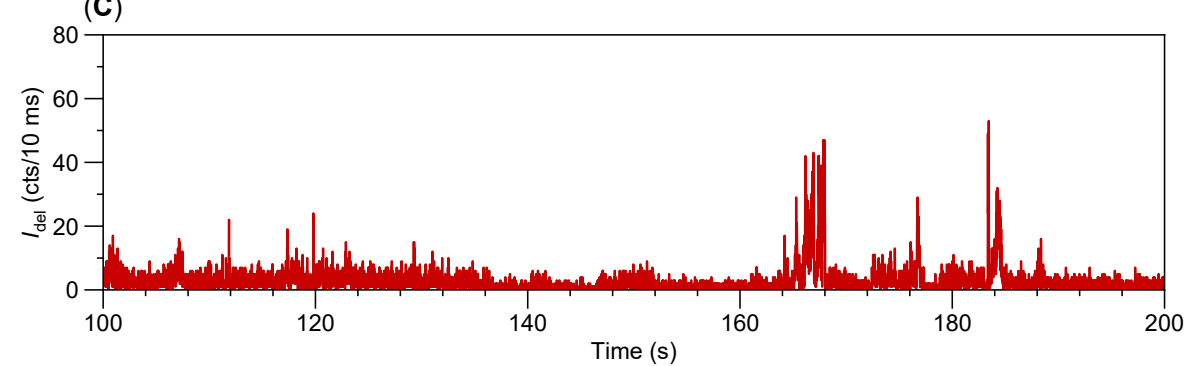

(E)
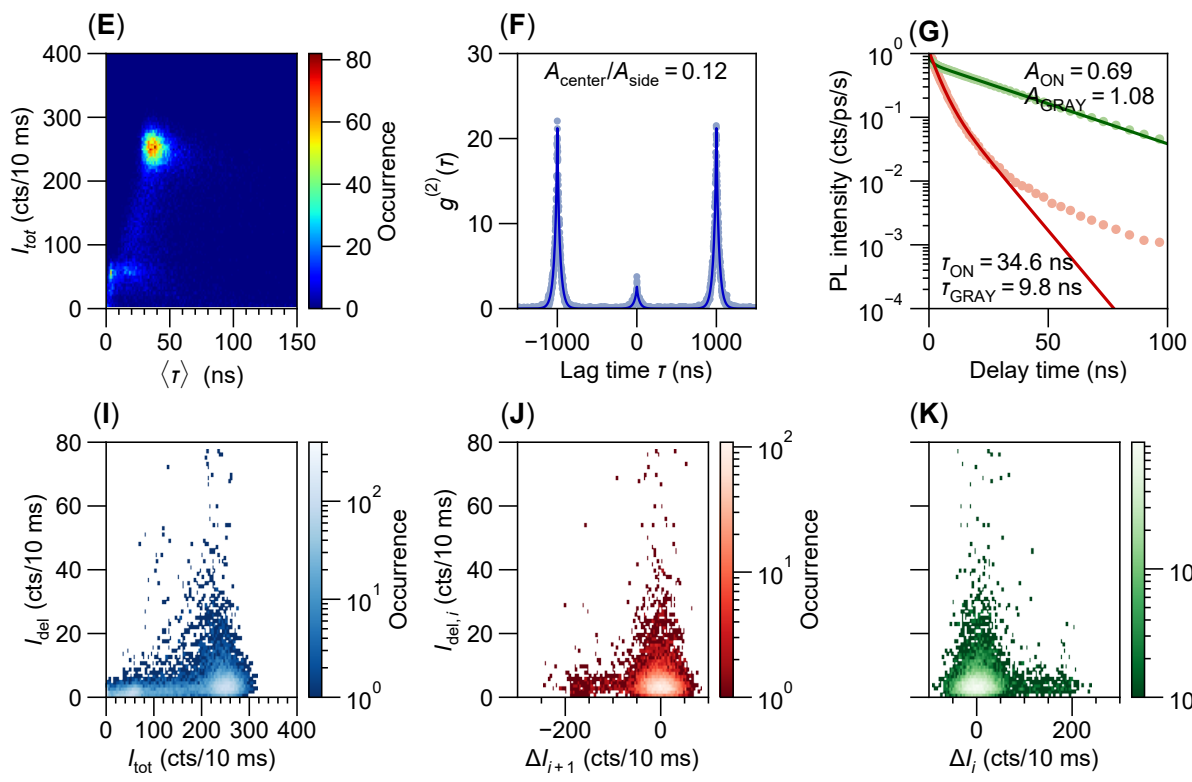

(K)

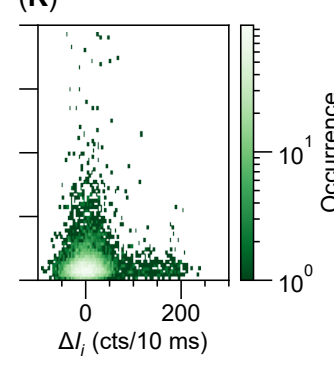

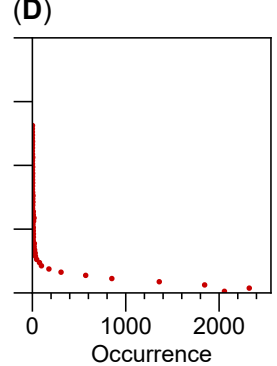

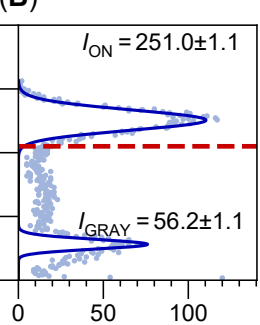

(D)

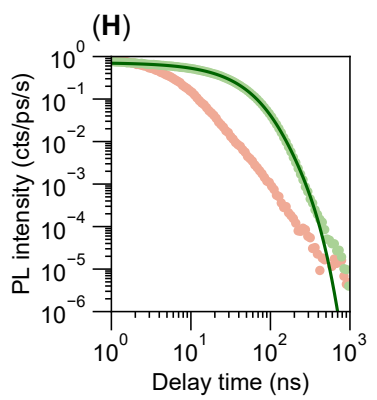

(L)

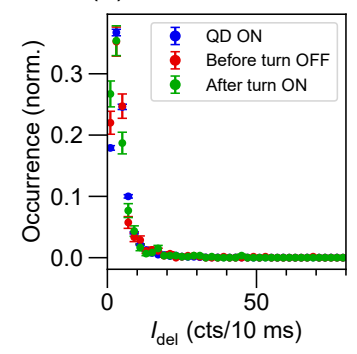

Figure E9 - Overview of photoluminescence properties of quantum dot 9. 


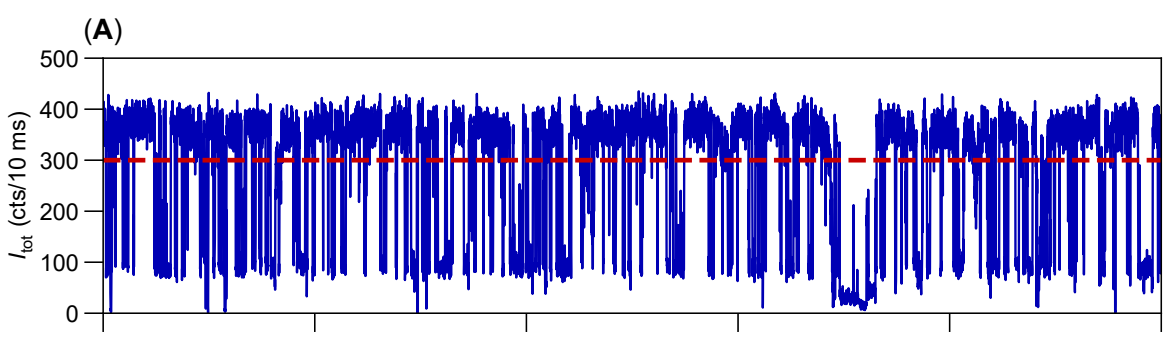

(C)

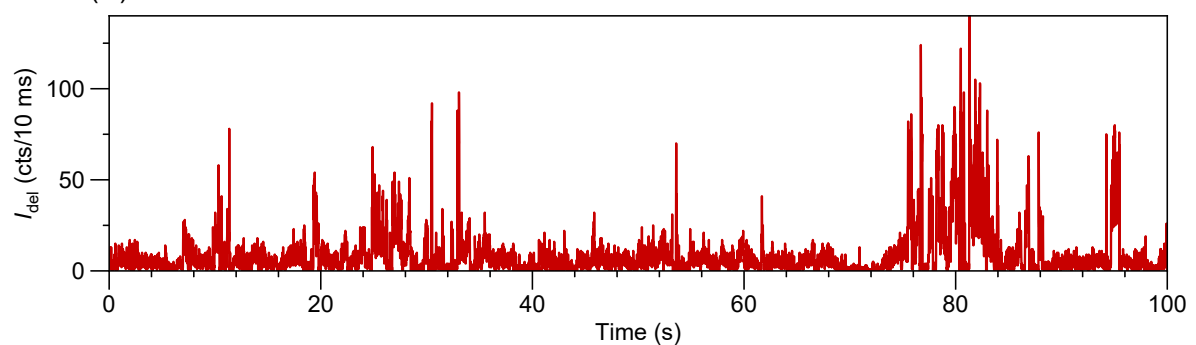

(E)

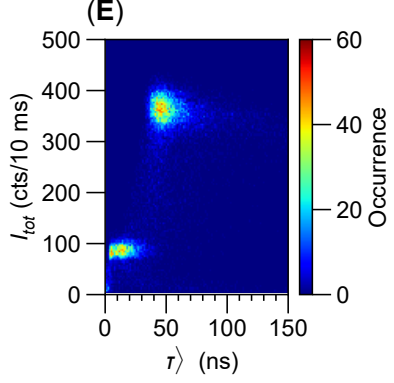

(I)

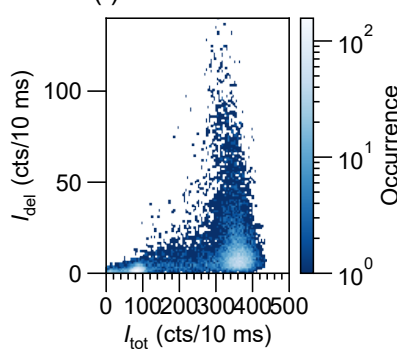

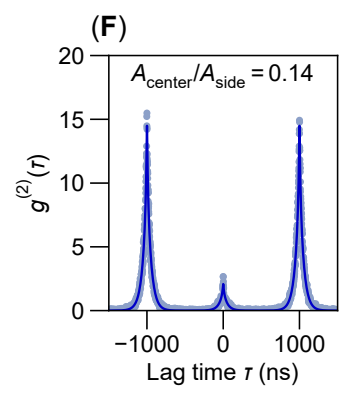

(J)

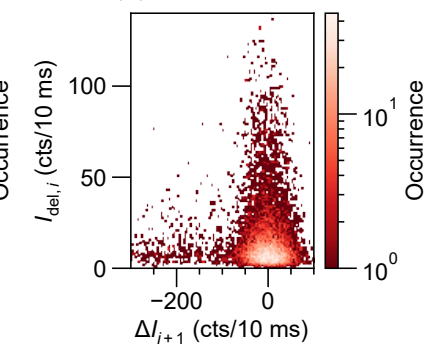

(B)

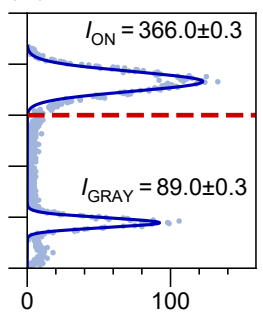

(D)

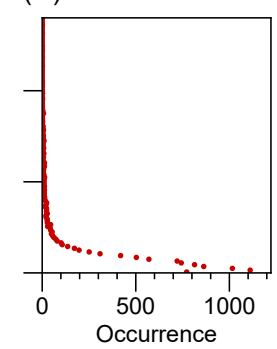

(H)

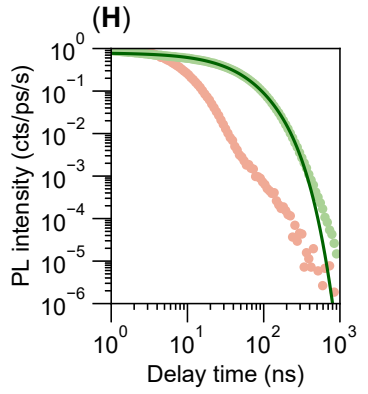

(L)

(K)

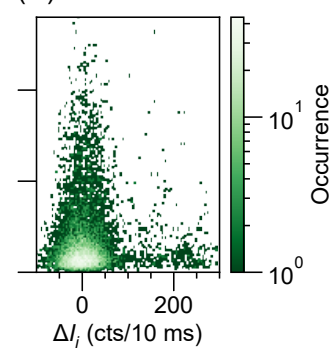

Figure E10 - Overview of photoluminescence properties of quantum dot 10. 
Figure E11-E20 - Results of spectrometer-APD experiments.

Below we provide an overview of results from experiments employing simultaneous detection by a single APD and a spectrometer. These experiments were performed directly after the double-APD experiments of Figure E1-E9.

The arrangement of each of these figures is as follows: (A) Distribution of delayed emission intensity, $I_{\text {del }}$. The colors (blue, cyan, green, yellow, orange, red) indicate the six delayed emission categories used in this work. (B) Photoluminescence decay curves from selected periods with increased delayed emission intensity, corresponding to the six delayed-emission categories indicated in (A). Symbols represent experimental data, lines are mono-exponential fits on delay-time range 50-150 ns. (C) The same as in (B), but displayed in double logarithmic axes. (D) Amplitudes and decay rates obtained from fits of exponential decay to the experimental decay curves depicted in (B). Symbols are experimental data, colors indicate the delayed-emission category (see A). The solid line is a linear fit to the data points. (E) Single-QD PL spectra as a function of $I_{\mathrm{del}}$, corresponding to the six delayed-emission categories indicated in (A). The dotted line is a guide to the eye and indicates the PL peak position of the 'no-delayed-emission' spectrum. (F) PL peak position and (G) full-width-at-half-maximum as a function of our selection of $I_{\text {del }}$, displayed here as the mean \pm standard deviation of Lorentzian fits to the selected 18-ms integrated spectral frames.

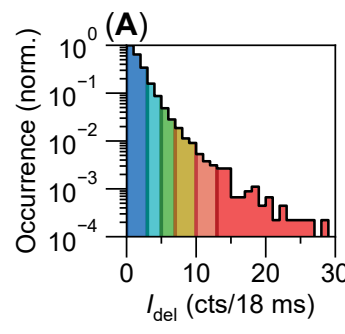

(B)

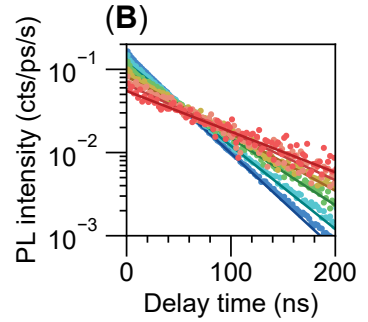

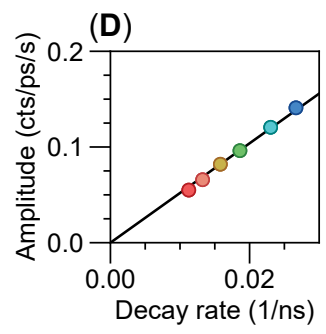

(C)

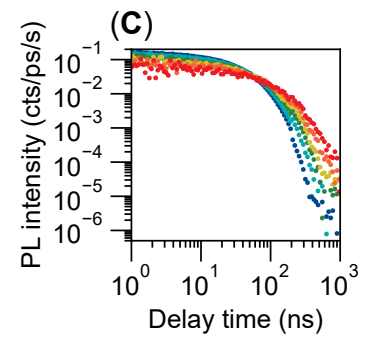

(E)

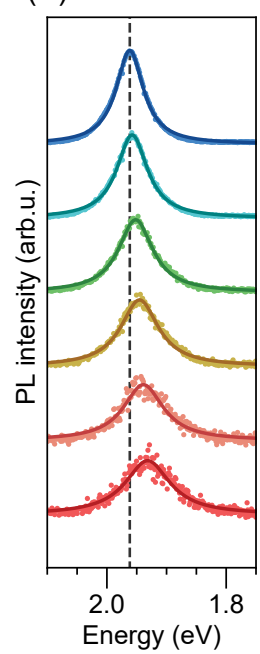

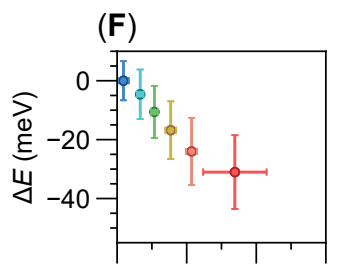

(G)

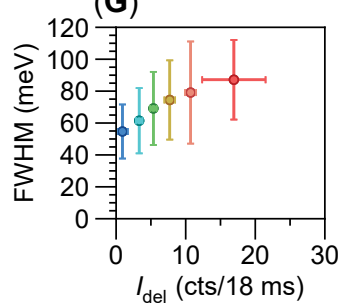

Figure E11 - Results of spectrometer-avalanche photon diode parallel measurements, quantum dot 1 (the quantum dot discussed in the main text). 

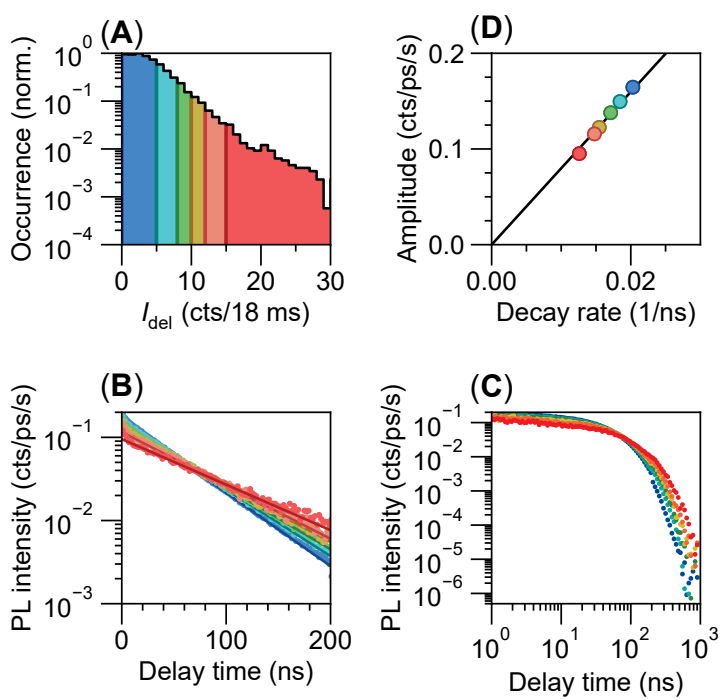

(E)

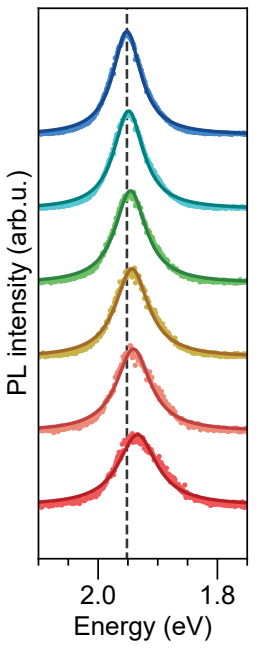

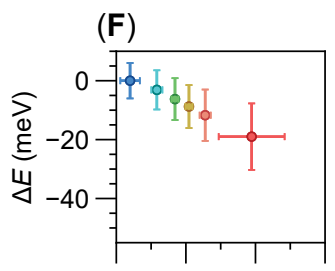

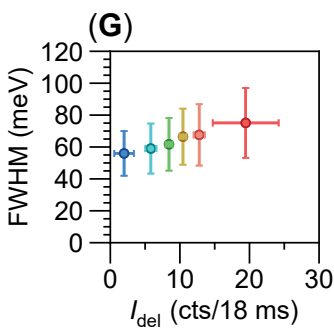

Figure E12 - Results of spectrometer-avalanche photon diode parallel measurements, quantum dot 2.
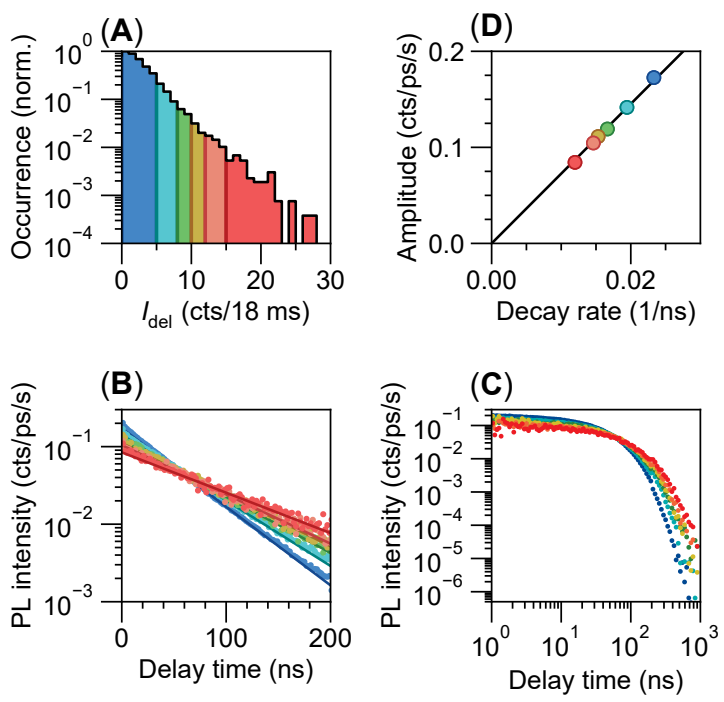

(E)

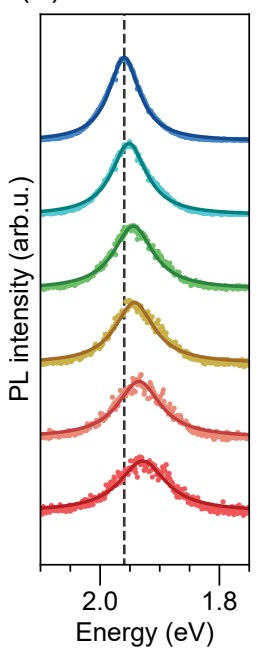

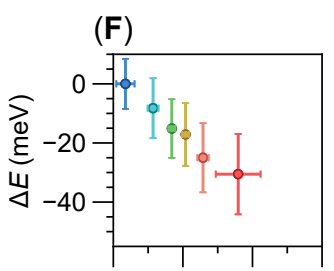

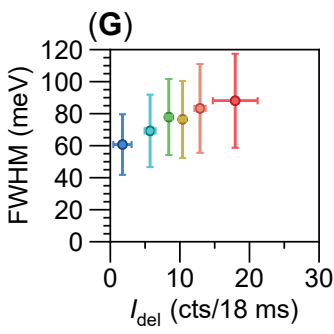

Figure E13 - Results of spectrometer-avalanche photon diode parallel measurements, quantum dot 3. 

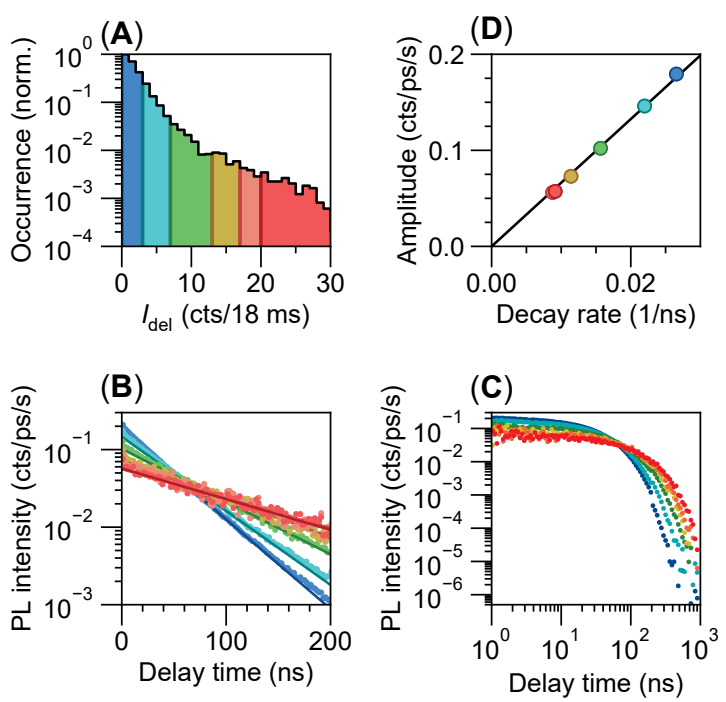

(E)

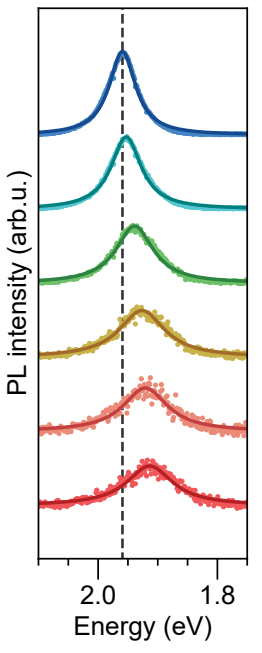

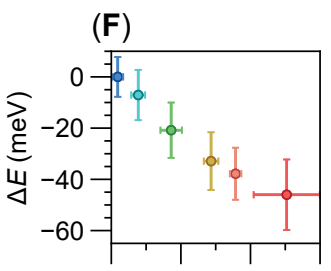

$(\mathbf{G})$

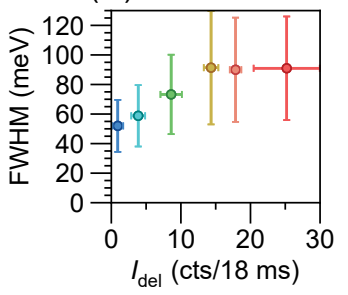

Figure E14 - Results of spectrometer-avalanche photon diode parallel measurements, quantum dot 4.
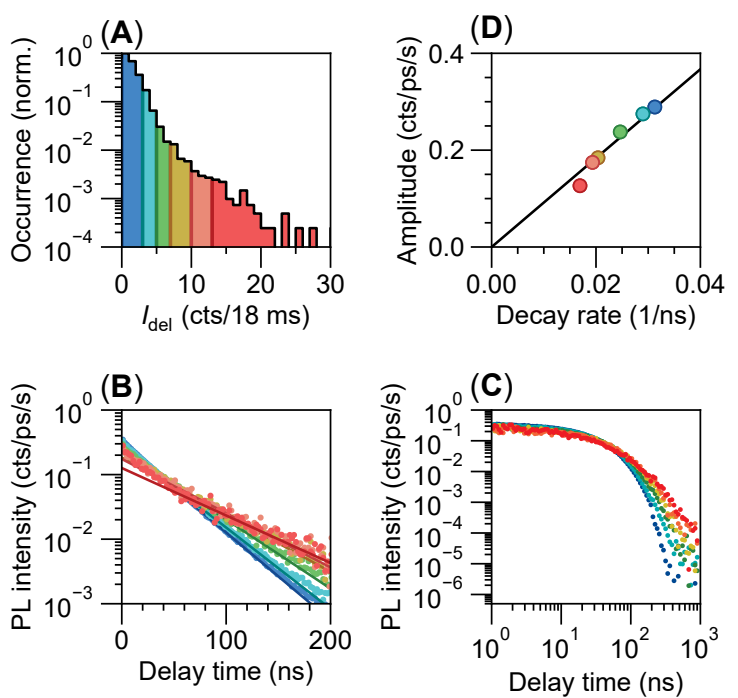

(E)

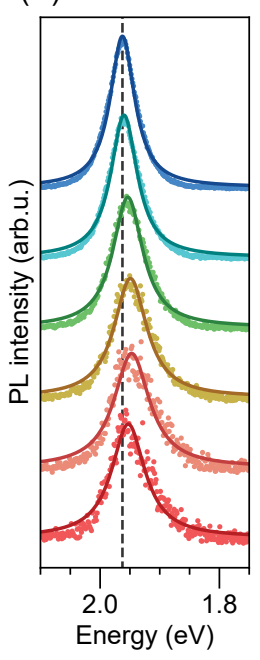

(F)
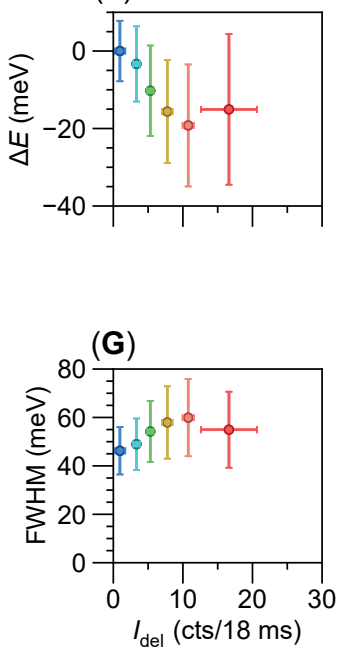

Figure E15 - Results of spectrometer-avalanche photon diode parallel measurements, quantum dot 5. 

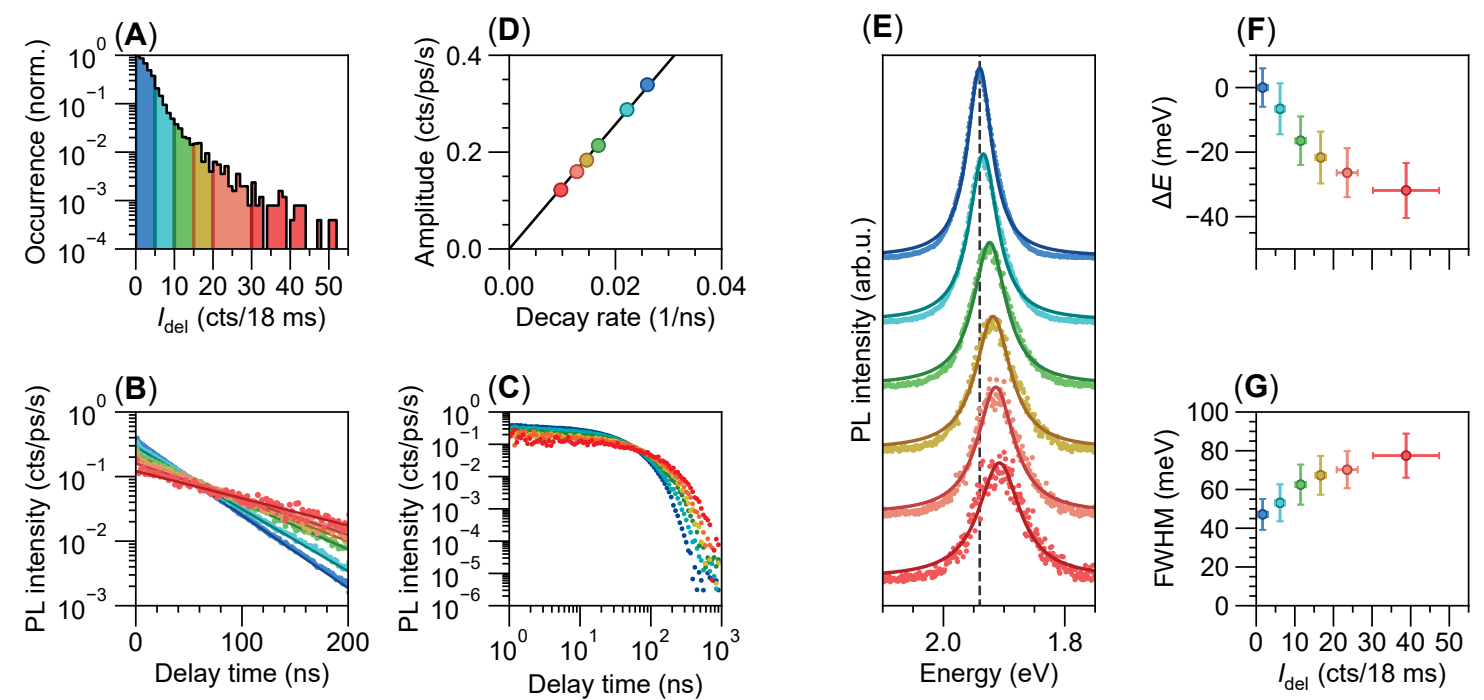

Figure E16 - Results of spectrometer-avalanche photon diode parallel measurements, quantum dot 6.
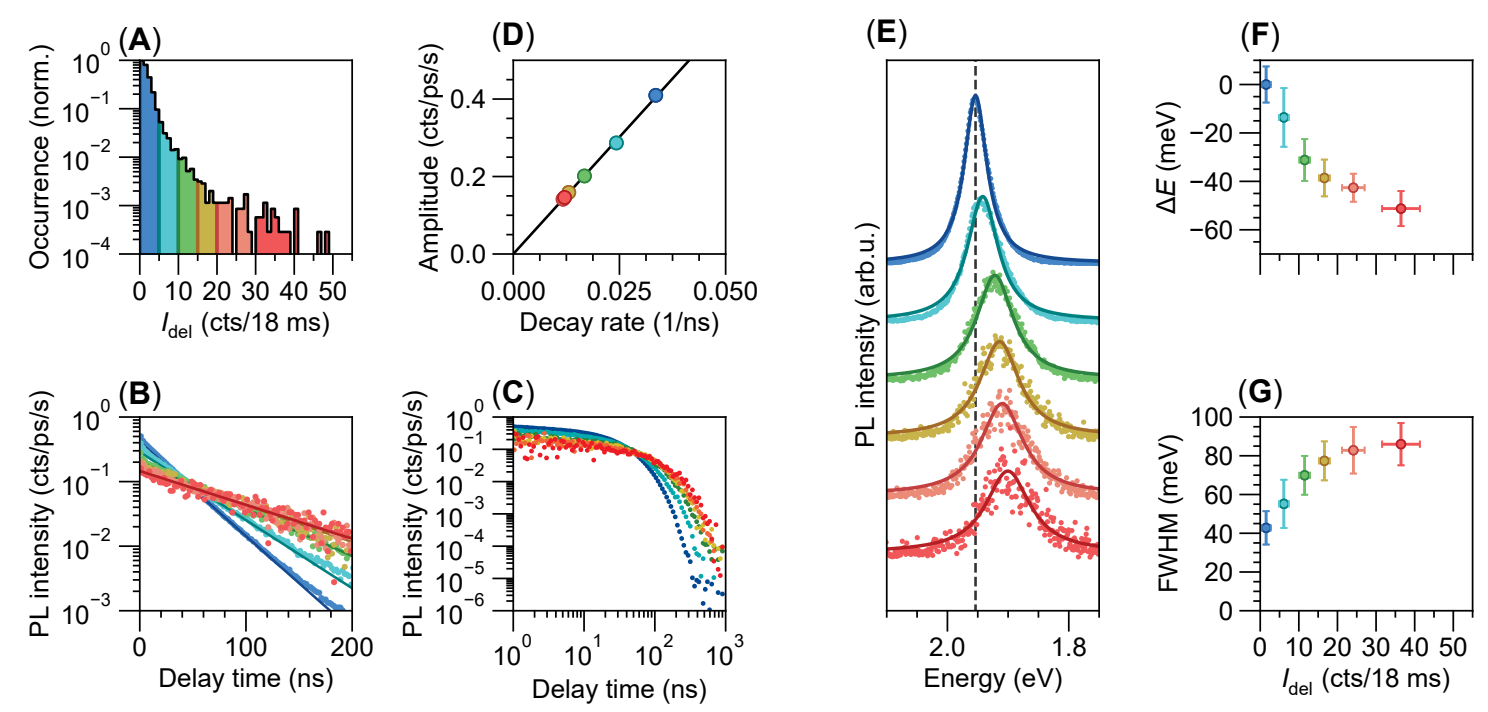

Figure E17 — Results of spectrometer-avalanche photon diode parallel measurements, quantum dot 7. 

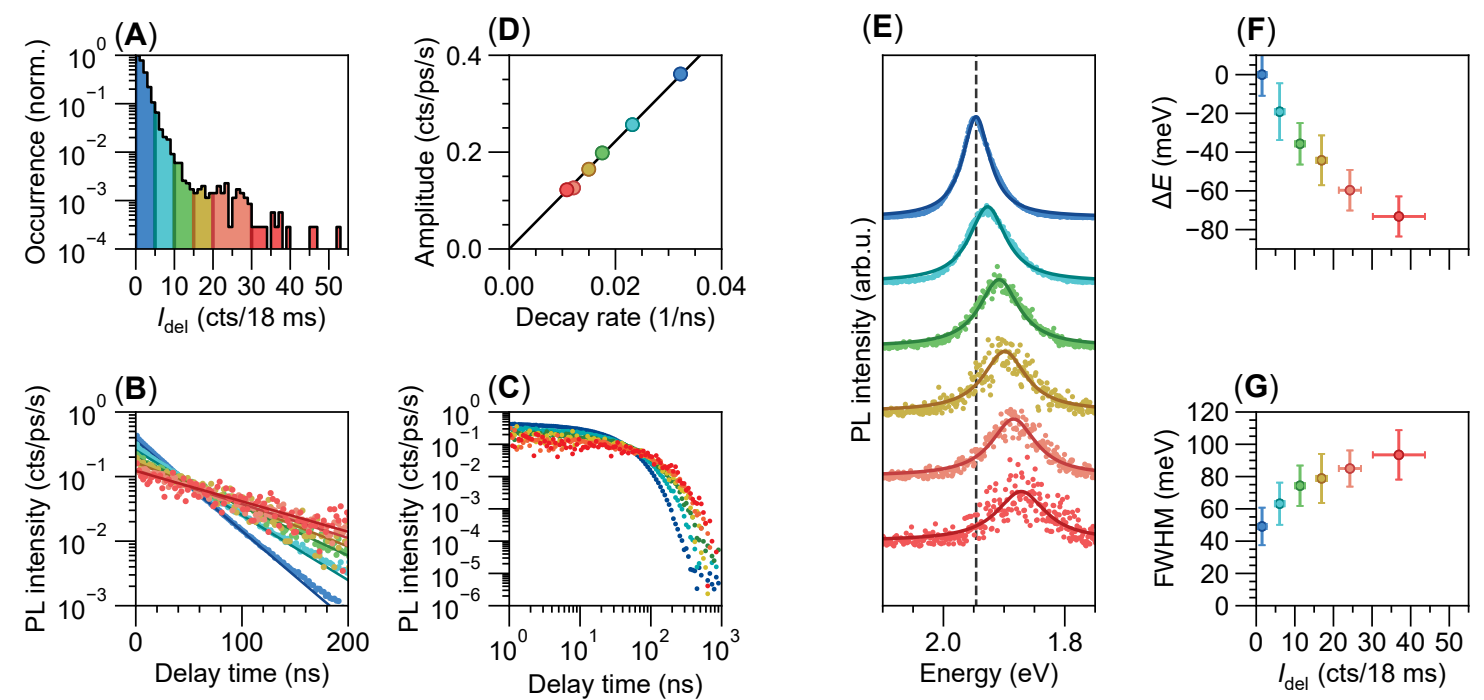

Figure E18 - Results of spectrometer-avalanche photon diode parallel measurements, quantum dot 8.
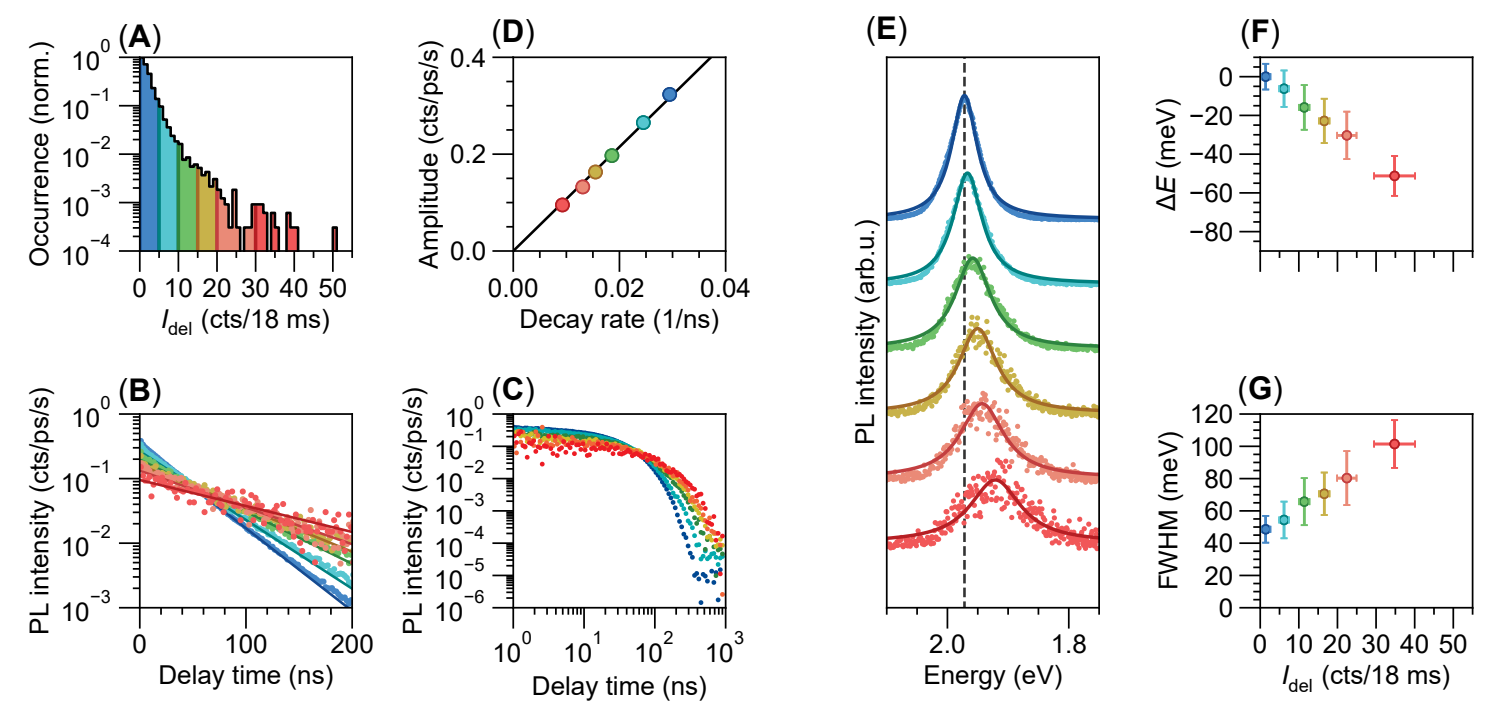

Figure E19 - Results of spectrometer-avalanche photon diode parallel measurements, quantum dot 9. 

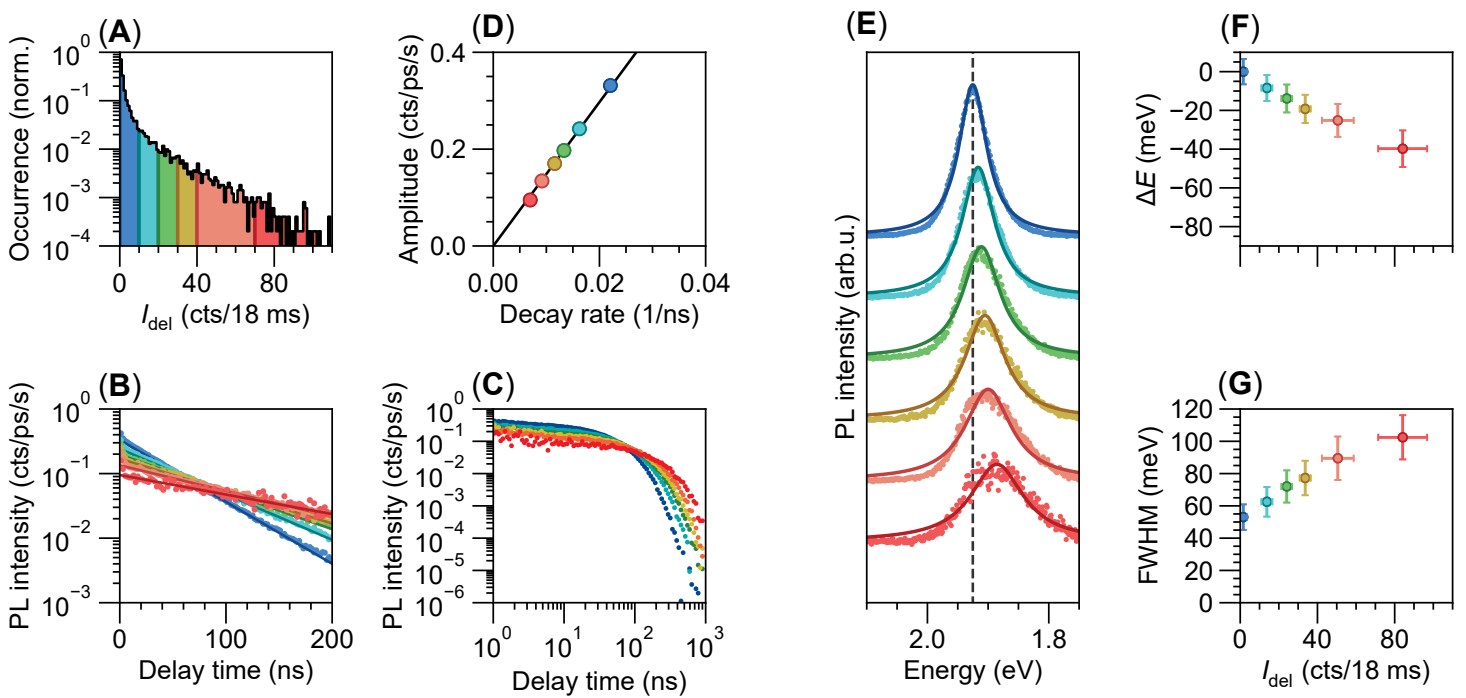

Figure E20 - Results of spectrometer-avalanche photon diode parallel measurements, quantum dot 10. 
